\title{
OSTEOTOMIA FEMORAL DISTAL DE VARIZAÇÃO PARA OSTEOARTROSE NO JOELHO VALGO: SEGUIMENTO EM LONGO PRAZO
}

\author{
DISTAL FEMORAL VARUSING OSTEOTOMY FOR OSTEOARTHRITIS \\ OF THE VALGUS KNEE: A LONG-TERM FOLLOW-UP
}

Marco Antônio Percope de Andrade', Davi Coutinho Fonseca Fernandes Gomes², André Lopes Portugal², Guilherme Moreira de Abreu e Silva ${ }^{3}$

\section{RESUMO}

Objetivo: Avaliar os resultados em longo prazo da osteotomia femoral distal de varização e procurar estabelecer critérios preditivos que possam auxiliar na seleção dos pacientes a serem submetidos a essa técnica. Métodos: Quinze pacientes portadores de osteoartrose do compartimento lateral e deformidade em valgo do joelho foram submetidos à osteotomia femoral distal de varização em "V" e fixação com placa-lâmina lateral, procurando-se alinhar o joelho a $0^{\circ}$ no eixo anatômico. O seguimento médio foi de 81,4 meses, variando de 43 a 132 meses. Utilizou-se o protocolo da Knee Society Rating System. Foram consideradas ainda as variáveis: idade do paciente, tempo de seguimento e ângulo anatômico pós-operatórios. Resultados: Foram obtidos 11 resultados excelentes e bons (73\%) e quatro regulares e ruins (27\%). Conclusão: A osteotomia femoral distal de varização em " $V$ " constitui boa opção para o tratamento de pacientes com osteoartrose do compartimento lateral e valgismo do joelho. Não se confirmaram as variáveis idade do paciente na data de cirurgia, tempo de seguimento e ângulo anatômico pós-operatórios como fatores preditivos dos resultados.

Descritores - Osteotomia; Osteoartrite; Joelho

\begin{abstract}
Objective: Assess the long-term results of distal femoral varusing osteotomy and try to establish predictive criteria that could help on selecting patients to be submitted to this technique. Methods: Fifteen patients with lateral compartment osteoarthritis and valgus deformity of the knee were submitted to distal femoral "V" varusing osteotomy fixated with lateral plate, pursuing knee alignment at $0^{\circ}$ on the anatomical axis. The mean follow-up period was 81.4 months, ranging from 43 to 132 months. The Knee Society Rating System protocol was employed. Additional assessed variables were the following: patient age, follow-up time, and postoperative anatomical angle. Results: 11 results were regarded as excellent or good (73\%) and four as fair or poor (27\%). Conclusion: Distal femoral "V" varusing osteotomy constitutes a good treatment alternative for patients with lateral compartment osteoarthritis and valgus knee. The following variables have not been confirmed: patient age at the time of surgery, follow-up time, and postoperative anatomical angle as predictive factors for the results.
\end{abstract}

Keywords - Osteotomy; Osteoarthritis; Knee

1 - Chefe do Serviço de Ortopedia e Traumatologia do Hospital das Clínicas da Universidade Federal de Minas Gerais; Professor Adjunto do Departamento do Aparelho Locomotor da Universidade Federal de Minas Gerais, Belo Horizonte - MG.

2 - Médico Residente do $3^{\circ}$ Ano do Programa de Ortopedia e Traumatologia do Hospital das Clínicas da Universidade Federal de Minas Gerais, Belo Horizonte - MG.

3 - Médico Ortopedista da Equipe de Joelho do Hospital das Clínicas da Universidade Federal de Minas Gerais, Belo Horizonte - MG.

Correspondência: Rua Maranhão, 1.567/701 - 30150-331 - Belo Horizonte, MG. Tel.: (31) 3273-5800. 


\section{INTRODUÇÃO}

A gonartrose, ou artrose degenerativa do joelho, evolui frequentemente com alteração do eixo do membro, em varo ou valgo, sendo este mais raro. No joelho varo a deformidade principal ocorre na tíbia, enquanto que no valgo o fêmur é mais acometido, levando à obliquidade da linha $\operatorname{articular}^{(1)}$.

A deformidade em valgo tende a evoluir para artrose mais tardiamente, sendo geralmente menos sintomática. Isso é explicado pelo momento adutor, que coloca a força da gravidade principalmente no compartimento medial durante a marcha, sendo necessária deformidade em valgo muito acentuada para que haja sobrecarga do compartimento lateral ${ }^{(2-4)}$. Esse momento adutor justifica também, biomecanicamente, a necessidade do alinhamento em $0^{\circ}$ do eixo anatômico nas osteotomias do joelho valgo, uma vez que, em condições fisiológicas, $60 \%$ do peso do corpo passam pelo compartimento medial ${ }^{(5,6)}$.

A indicação de osteotomia para o tratamento da gonartrose vem se tornando mais restrita em virtude da grande melhora nos resultados em longo prazo da artroplastia total ${ }^{(7)}$. No entanto, ela tem a grande vantagem de não ser um procedimento substitutivo, o que é mais relevante em pacientes jovens. A osteotomia femoral é mais eficaz que a tibial para se abordar a deformidade em valgo do joelho, por tornar a linha transcondiliana perpendicular ao eixo mecânico e minimizar o afrouxamento do ligamento colateral medial ${ }^{(1,8,9)}$.

O objetivo deste estudo é avaliar os resultados em longo prazo da osteotomia femoral distal de varização e procurar estabelecer critérios preditivos que possam auxiliar na seleção dos pacientes a serem submetidos a essa técnica.

\section{MÉTODOS}

No período de julho de 1997 a novembro de 2004 foram realizadas 23 osteotomias de varização do fêmur distal para tratamento da gonartrose, pelo mesmo cirurgião, na técnica descrita no presente artigo. Foram excluídos do estudo oito pacientes, sendo seis por não ter sido localizados, um por ter falecido antes do início da avaliação e um por não apresentar condições clínicas de avaliação funcional devido a um câncer de cólon terminal. O grupo final do estudo, portanto, foi composto de 15 pacientes submetidos à osteotomia de varização do fêmur distal e fixação com placa-lâmina. Dos pacientes, 13 apresentavam gonartrose lateral primária e dois, pós-traumática.
Os pacientes foram categorizados de acordo com a idade (menos de 20, de 20 a 40, de 40 a 60 e acima de 60 anos), tempo de seguimento pós-operatório (menos de 50 , de 50 a 100 e mais de 100 meses) e ângulo anatômico pós-operatório (mais de $2^{\circ}$ de varo, de $2^{\circ}$ de varo a $3^{\circ}$ de valgo e mais de $3^{\circ}$ de valgo) .

A idade dos pacientes na época da cirurgia variou de 19 a 72 anos (média de 49,8). Três eram do sexo masculino e 12 do feminino. O seguimento pós-operatório variou de 43 a 132 meses (média de 81,4). Foram realizadas 10 osteotomias no joelho direito e cinco no esquerdo. Nenhum paciente foi submetido à osteotomia bilateral do fêmur. Uma paciente foi submetida previamente à osteotomia de valgização da tíbia contralateral e outra, ao realinhamento patelar contralateral.

\section{Avaliação pré-operatória e indicação do procedimento}

$\mathrm{Na}$ avaliação pré-operatória os pacientes foram submetidos a entrevista pessoal e exame clínico ortopédico pelo autor sênior, sendo realizadas radiografias dos joelhos na incidência ântero-posterior (AP) em ortostatismo, com apoio monopodálico, em filme 30x40 cm para medição do ângulo anatômico, ou ângulo femorotibial, respeitando-se a mesma posição rotacional dos membros inferiores a partir da observação da posição da patela e dos pés no momento do exame ${ }^{(10-15)}$ (Figura 1). As incidências em perfil do joelho e axial de patela foram utilizadas para confirmar o diagnóstico de artrose unicompartimental lateral.

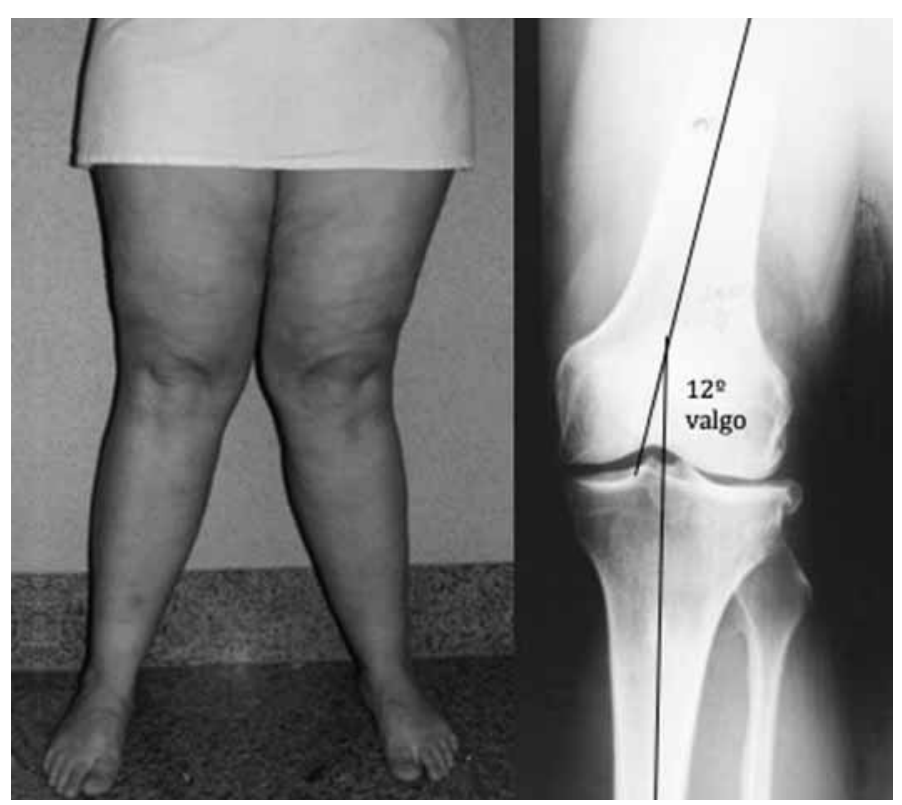

Figura 1 - Avaliação clínica e radiográfica pré-operatória com traçado do eixo anatômico 


\section{Técnica cirúrgica}

Através de um acesso lateral ao fêmur distal, foram realizadas osteotomia supracondiliana em "V"(15-18) de $45^{\circ}$ e osteossíntese com placa- lâmina lateral em todos os 15 pacientes. Para se obter o alinhamento neutro do eixo

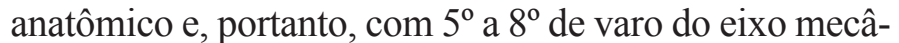
nico ${ }^{(12,14,15,17,19,20)}$, a lâmina da placa de ângulo fixo em $95^{\circ}$ foi inserida paralela ou com angulação de até $5^{\circ}$ de varo em relação à superfície articular da tíbia sob visualização radioscópica. A placa foi então fixada ao fêmur após verificação do eixo mecânico por meio de fio metálico posicionado externamente do centro da cabeça do fêmur ao centro da articulação do tornozelo ${ }^{(21)}$ (Figura 2).

No pós-operatório imediato foram iniciados exercícios isométricos e isotônicos de flexoextensão ativa do joelho sob supervisão fisioterápica. A descarga de peso no membro operado foi permitida após seis a oito semanas, de acordo com sinais clínicos e radiográficos de consolidação da osteotomia.

\section{Avaliação pós-operatória em longo prazo}

Para avaliação pós-operatória em longo prazo, subjetiva e objetiva, os pacientes retornaram à instituição para entrevista pessoal com os autores do estudo, realização de exame clínico ortopédico e avaliação radiográfica do joelho operado (Figura 3). Foi então aplicado o questionário padronizado Knee Society Rating System $(K S S)^{(22)}$, modificado no critério alinhamento para se adequar às finalidades do estudo. O KSS originalmente foi elaborado para avaliação de pacientes submetidos à artroplastia de joelho na qual se objetiva o alinhamento mecânico em neutro, utilizando os seguintes critérios para pontuação referente ao alinhamento: angulação final entre $5^{\circ}$ e $10^{\circ}$ de valgo, não é feita dedução no escore; entre $0^{\circ}$ e $4^{\circ}$ ou entre $11^{\circ}$ e $15^{\circ}$ de valgo, são deduzidos três pontos para cada grau; e para angulações em varo ou maiores do que $15^{\circ}$ de valgo, são deduzidos 20 pontos. Já no presente estudo, nos casos com angulação final entre $2^{\circ}$ de varo

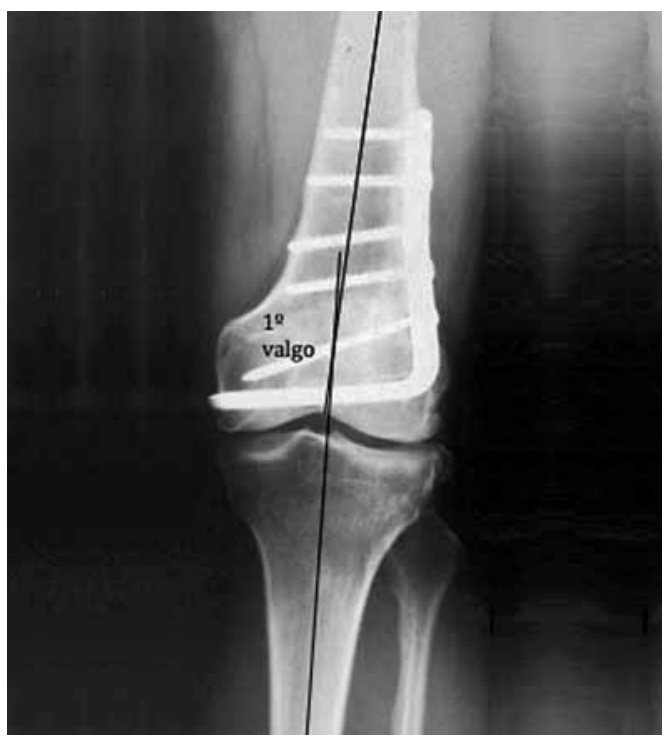

Figura 3 - Avaliação radiográfica pós-operatória com traçado do eixo anatômico desejável

e $3^{\circ}$ de valgo, não foi feita dedução no escore por se considerar o resultado pretendido para o procedimento $^{(12,16,17,23,24)}$. Foram deduzidos três pontos para cada grau entre 3 e $7^{\circ}$ de varo ou entre 4 e $8^{\circ}$ de valgo, e 20 pontos para angulações maiores que $7^{\circ}$ de varo ou $8^{\circ}$ de valgo.

$\mathrm{Na}$ avaliação radiográfica foram utilizadas a incidência AP em ortostatismo com apoio monopodálico em filme $30 x 40 \mathrm{~cm}$ para medição do ângulo anatômico e a incidência em perfil do joelho para avaliação do alinhamento em neutro da osteotomia no plano sagital.

\section{Análise estatística}

Com o objetivo de avaliar a existência de relação estatisticamente significativa entre os resultados excelentes/bons e regulares/ruins obtidos pelo protocolo KSS com as variáveis idade dos pacientes na data da cirurgia, tempo de seguimento pós-operatório e ângulo anatômico pós-operatório, foi realizada análise estatística pelo teste exato de Fisher após categorização das variáveis. Utilizou-se valor de $\mathrm{p}<0,05$ para significância estatística.
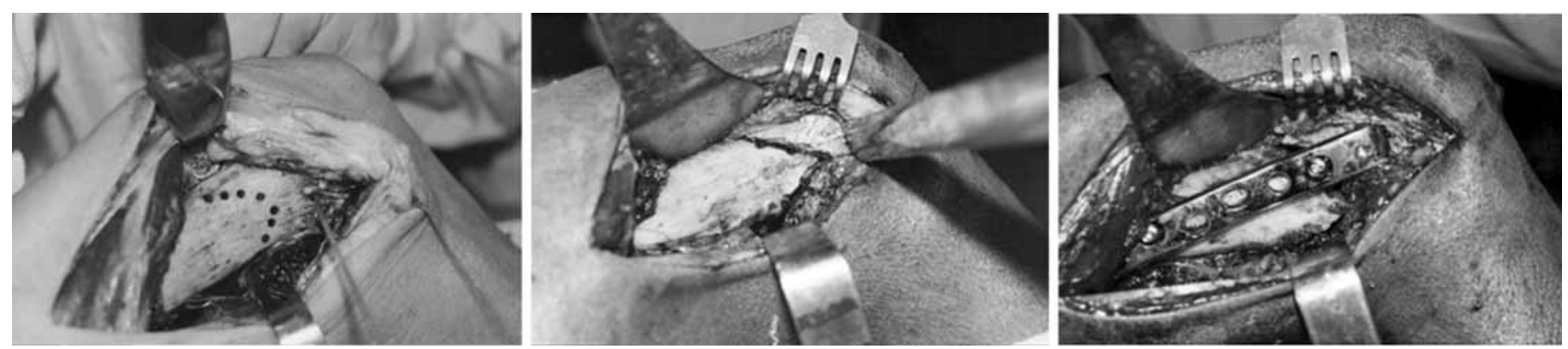

Figura 2 - Técnica operatória: A) Acesso medial, fios-guia para orientação do posicionamento da placa-lâmina, marcação da osteotomia em "V"; B) Osteotomia em "V" e fresagem para inserção da placa lâmina; C) Placa-lâmina fixada, sendo vista a translação lateral do fragmento distal da osteotomia 


\section{RESULTADOS}

Segundo o KSS, foram obtidos cinco resultados excelentes (acima de 170 pontos), seis bons (entre 140 e 170 pontos), um regular (entre 125 e 139 pontos) e três ruins (abaixo de 125 pontos). Os resultados excelentes e bons somaram $73 \%$ e os regulares e ruins, $27 \%$ do total (Figura 4).

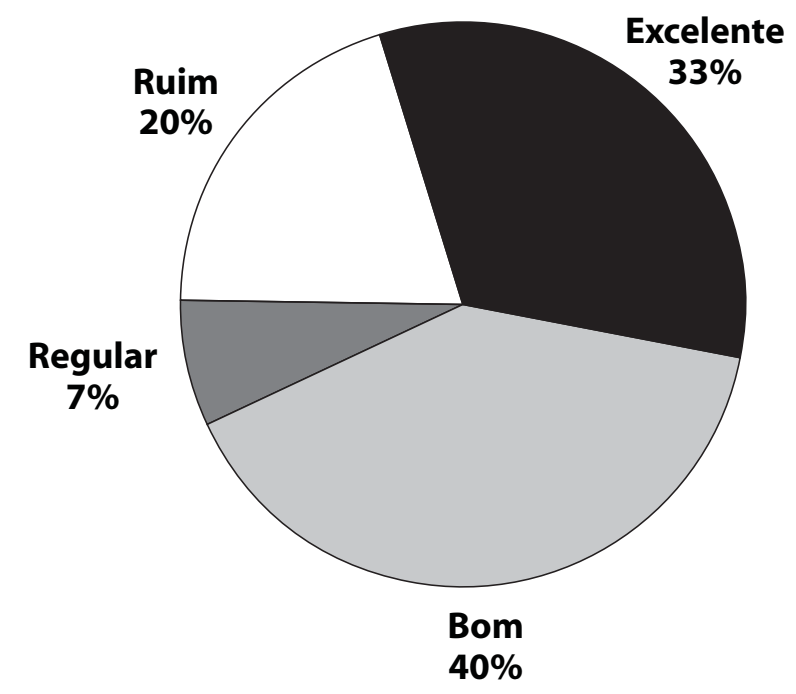

Figura 4 - Distribuição percentual dos resultados obtidos pelo Knee Society Rating System (KSS)

A variável ângulo anatômico pós-operatório final variou de $11^{\circ}$ de varo a $11^{\circ}$ de valgo (média de $1,73^{\circ}$ de valgo); sete pacientes (46\%) permaneceram na faixa pretendida de $2^{\circ}$ de varo a $3^{\circ}$ de valgo.

Ao se categorizar a variável idade em quatro faixas etárias (menos de 20, de 20 a 40, de 40 a 60 e mais de 60 anos), não houve diferença estatisticamente significativa entre os grupos de resultados excelentes/bons e regulares/ruins $(\mathrm{p}=0,73)$.

Ao se considerar a variável tempo de seguimento pós-operatório em três períodos (menos de 50 , de 50 a 100 e mais de 100 meses), não houve diferença estatisticamente significativa entre os grupos de resultados excelentes/bons e regulares/ruins $(p=0,58)$.

Dividindo-se o ângulo pós-operatório em três grupos de valores (mais de $2^{\circ}$ de varo, de $2^{\circ}$ de varo a $3^{\circ}$ de valgo e mais de $3^{\circ}$ de valgo), não houve diferença estatisticamente significativa entre os grupos de resultados excelentes/bons e regulares/ruins $(p=0,50)$.

\section{DISCUSSÃO}

A osteotomia femoral distal de varização está indicada na artrose unicompartimental lateral que apresenta ângulo anatômico maior do que $12^{\circ}$ de valgo ou inclinação da superfície articular do joelho maior do que $10^{\circ}$ no plano coronal em relação à horizontal ${ }^{(12,13,15,17,23-25)}$. O procedimento é contra-indicado em pacientes com dor inespecífica no joelho, artrose ou meniscectomia prévia no compartimento medial e doença inflamatória. Idade acima de 60 anos, obesidade, artrose grave, arco de movimento do joelho menor do que $90^{\circ}$ e instabilidade ligamentar do joelho moderada ou grave são consideradas contra-indicações relativas ${ }^{(1,12,13,15)}$. A artrose femoropatelar grave, caracterizada por dor anterior predominante e sinais radiográficos de osteófitos e pinçamento articular, foi considerada contra-indicação absoluta para a osteotomia femoral, embora em estudo recente de Wang e $\mathrm{Hsu}^{(26)}$ não tenha havido diferença no resultado da osteotomia de oito pacientes com artrose femoropatelar grave em relação aos casos de artrose unicompartimental.

A medida do eixo anatômico na radiografia em AP com apoio monopodálico foi empregada por ser de mais fácil execução e de menor custo quando comparada com utilização da medida do eixo mecânico ${ }^{15}$, considerandose ainda que, independente da técnica de medição do eixo, não há correlação precisa entre os achados radiográficos (estáticos) e a distribuição de carga (dinâmicos), mesmo após osteotomia corretiva ${ }^{(3,4)}$.

Os resultados da osteotomia de varização do fêmur para tratamento do joelho valgo descritos na literatura são bastante variados, refletindo a influência de fatores como seleção do paciente, técnica cirúrgica, alinhamento pós-operatório e tempo de seguimento ${ }^{(8,20,27,28)}$. Desses fatores, a influência do alinhamento pós-operatório sobre a evolução clínica é bem documentada ${ }^{(12,16,17,23,24)}$. Embora ainda não tenham sido realizados estudos clínicos prospectivos, o alinhamento com um ângulo femorotibial de $0^{\circ}$ é geralmente aceito como a correção pósoperatória pretendida ${ }^{(24)}$. O presente estudo considerou como bom alinhamento o eixo entre $2^{\circ}$ de varo e $3^{\circ}$ de valgo, baseando-se na modificação da faixa de bom alinhamento do KSS para se adequar ao alinhamento anatômico em neutro pretendido após o procedimento. Não se observou associação estatisticamente significativa entre o ângulo anatômico final e os resultados do escore KSS, embora três dos quatro resultados regulares/ruins tenham ocorrido nos pacientes com ângulos anatômicos finais $\left(10^{\circ}\right.$ de varo, $10^{\circ}$ de valgo e $11^{\circ}$ de valgo) mais distantes da faixa considerada ideal. 
O número de pacientes do estudo $(\mathrm{n}=15)$ é pequeno, porém comparável com o da literatura referente à osteotomia de varização do fêmur distal pela técnica em "V" que, nos artigos avaliados, variou de 11 a 17, com média de $13,7^{(15-18)}$.

O tempo de seguimento pós-operatório neste estudo (81,4 meses) foi maior do que o da maioria da literatura. De 18 estudos avaliados $(8,10,11,14-19,23,24,26,27,29-33)$, apenas seis $^{(10,11,17,26,29,32)}$ apresentaram tempo médio superior.

A faixa etária entre 19 e 72 anos (média de 49,8 anos) também foi similar à dos outros estudos, sendo observada, na literatura, variação de 14 a 79 anos, com média entre 34,8 e $58 \operatorname{anos}^{(14-18,23,27,30,31,33)}$.

A comparação dos resultados obtidos neste estudo pela aplicação do KSS é difícil pela grande variedade metodológica encontrada na literatura. No entanto, ao se compararem esses dados, pôde-se constatar que o índice de resultados excelentes e bons (73\%) foi superior aos de seis ${ }^{(8,10,11,15,27,31)}$ dos estudos, com índices entre 57,1 e $71,4 \%$. Dos 12 estudos ${ }^{(14,16-19,23,24,26,29,30,32,33)}$ com resultados superiores (índices entre 76,4 e 100\%), apenas quatro ${ }^{(17,26,29,32)}$ tinham seguimento mais longo. Esse dado mostra-se relevante na medida em que é descrita na literatura deterioração dos resultados ao longo do tempo de seguimento $^{(6,10,26,30)}$. Não obstante, Edgerton et $a^{(11)}$ não demonstraram essa tendência, assim como este estudo.

Comparando-se os resultados apenas com os estudos de osteotomia em "V", puderam-se observar índices de sucesso semelhantes. Aglietti et $a l^{(15)}$ apresentaram $71,4 \%$ de resultados excelentes e bons em um grupo de 14 pacientes, após seguimento médio de 21,8 meses. Em 1991, Cerqueira et al ${ }^{(16)}$ obtiveram $81,8 \%$ de resultados excelentes e bons em 11 pacientes, com seguimento médio de 42 meses. Costa et al ${ }^{(18)}$ avaliaram 13 pacientes, com seguimento médio de 24 meses, e apresentaram $76,9 \%$ de resultados satisfatórios. Aglietti e Menchetti ${ }^{(17)}$, após seguimento médio de 108 meses de 17 pacientes, obtiveram $76,4 \%$ de resultados excelentes e bons.

\section{CONCLUSÃO}

Concluiu-se que a osteotomia femoral distal de varização em "V" constitui boa opção para o tratamento de pacientes com osteoartrose do compartimento lateral e valgismo do joelho. No entanto, não se confirmaram como fatores preditivos dos resultados as variáveis idade do paciente na data de cirurgia, tempo de seguimento e ângulo anatômico pós-operatórios.

\section{REFERÊNCIAS}

1. Leone JM, Hansen AD. Osteotomy about the knee: american perspective. In: Scott WN, et al. Insall \& Scott surgery of the knee. 4th ed. New York: Churchill Livingstone; 2006. p. 1301-14

2. Andriacchi TP. Dynamics of knee malalignment. Orthop Clin North Am. 1994;25(3):395-403.

3. Harrington IJ. Static and dynamic loading patterns in knee joints with deformities. J Bone Joint Surg Am. 1983;65(2):247-59.

4. Johnson F, Leitl S, Waugh W. The distribution of load across the knee. A comparison of static and dynamic measurements. J Bone Joint Surg Br. 1980;62(3):346-9.

5. Franco V, Cerullo G, Cipolla M, Gianni E, Puddu G. Open wedge high tibial osteotomy. Techn Knee Surg. 2002;1(1):43-53.

6. Poilvache P. Osteotomy for the arthritic knee: a european perspective. In: Scott WN, et al. In: Scott WN, editor. Surgery of the Knee. 4th ed. New York: Churchill Livingstone; 2006. p. 1351-8.

7. Crockarell JR Jr, Guyton JL. Artroplastia de tornozelo e joelho. In: Canale ST. Cirurgia ortopédica de Campbell. 10a. ed. Barueri, SP: Manole; 2006. p. 245-51.

8. Mathews J, Cobb AG, Richardson S, Bentley G. Distal femoral osteotomy for lateral compartment osteoarthritis of the knee. Orthopedics. 1998;21(4):437-40.

9. Jackson JP. Osteotomy for osteoarthritis of the knee. Proceedings of the Sheffield Regional Orthopaedic Club. J Bone Joint Surg Br. 1958;40:826.

10. Finkelstein JA, Gross AE, Davis A. Varus osteotomy of the distal part of the femur. A survivorship analysis. J Bone Joint Surg Am. 1996;78(9):1348-52.

11. Edgerton BC, Mariani EM, Morrey BF. Distal femoral varus osteotomy for painful genu valgum. A five-to-11-year follow-up study. Clin Orthop Relat Res. 1993;(288):263-9.

12. Coventry MB. Osteotomy about the knee for degenerative and rheumatoid arthritis. J Bone Joint Surg Am. 1973;55(1):23-48.

13. Coventry MB. Current concepts review: upper tibial osteotomy for osteoarthritis. J Bone Joint Surg Am. 1985;67(7):1136-40.

14. Queiroz AAB, Navarro RD, Kubota MS. Correção da deformidade em valgo do joelho através da osteotomia cuneiforme de subtração supracondiliana do fêmur e utilização simultânea de enxerto autológo do ilíaco. Rev Bras Ortop 1993;28(5):258-62.

15. Aglietti P, Stringa G, Buzzi R, Pisaneschi A, Windsor RE. Correction of valgus knee deformity with a supracondylar V osteotomy. Clin Orthop Relat Res. 1987;(217):214-20.

16. Cerqueira NB, Souza JMB, Mendes ML. Osteotomia frontal supracondiliana do fêmur em "V" no perfil. Nova técnica para tratamento da deformidade em valgo do joelho. Rev Bras Ortop. 1991;26(10):365-8.

17. Aglietti P, Menchetti PP. Distal femoral varus osteotomy in the valgus osteoarthritic knee. Am J Knee Surg. 2000;13(2):89-95

18. Costa RA, Santos HRO, Meneses LC. Osteotomia supracondiliana do fêmur em "V" no tratamento da deformidade em valgo do joelho. Rev Bras Ortop. 1992;27(4):222-6.

19. Learmonth ID. A simple technique for varus supracondylar osteotomy in genu valgum. J Bone Joint Surg Br. 1990;72(2):235-7.

20. Maquet $P$. The treatment of choice in osteoarthritis of the knee. Clin Orthop Relat Res. 1985;192:108-12

21. Dietrick TB, Bugbee WD. Distal femoral osteotomy utilizing a lateral opening-wedge technique. Techn Knee Surg. 2005;4(3):186-92.

22. Insall JN, Dorr LD, Scott RD, Scott WN. Rationale of the Knee Society clinical rating system. Clin Orthop Relat Res. 1989;(248):13-4.

23. Healy WL, Anglen JO, Wasilewski SA, Krackow KA. Distal femoral varus osteotomy. J Bone Joint Surg Am. 1988;70(1):102-9.

24. McDermott AG, Finklestein JA, Farine I, Boynton EL, Maclntosh DL, Gross A. Distal femoral varus osteotomy for valgus deformity of the knee. J Bone Joint Surg Am. 1988;70(1):110-6.

25. Coventry MB. Proximal tibial varus osteotomy for osteoarthritis of the lateral compartment of the knee. J Bone Joint Surg Am. 1987; 69(1):32-8

26. Wang J, Hsu C. Distal femoral varus osteotomy for osteoarthritis of the knee. Surgical technique J Bone Joint Surg Am. 2006;(88 Suppl 1):100-8.

27. Terry GC, Cimino PM. Distal femoral osteotomy for valgus deformity of the knee. Orthopedics. 1992;15(11):1283-9.

28. Johnson EW Jr, Bodell LS. Corrective supracondylar osteotomy for painful genu valgum. Mayo Clin Proc. 1981;56(2):87-92.

29. Cameron HU, Botsford DJ, Park YS. Prognostic factors in the outcome of supracondylar femoral osteotomy for lateral compartment osteoarthritis of the knee. Can J Surg. 1997; 40(2):114-8.

30. Oliveira LP, Elias N, Cunha S, Mesquita KC. Osteotomia varizante distal do fêmur no tratamento do joelho valgo associado a artrose unicompartimental. Rev Bras Ortop. 1993;28(11/12):85-8.

31. Severino NR, Camargo OPA, Aihara T, Cury RPL, Oliveira VM, Barbi L, Medeiros SF et al. Osteotomia supracondiliana femoral no tratamento da deformidade em valgo do joelho. Rev Bras Ortop. 1998;33(4):282-6.

32. Cognet JM, Rouvillain JL, Mousselard HP. Résultat des ostéotomies fémorales de varisation pour genu valgum: a propos de 75 cas revus à plus de cinq ans de recul. Rev Chir Orthop Reparatrice Appar Mot. 1998;84:46.

33. Navarro RD, Laredo Filho J. Correção da deformidade em valgo do joelho, por via de acesso anterior, pela osteotomia de subtração supracondiliana do fêmur. Rev Bras Ortop. 1992;27(4):217-21 